\title{
MODULAR FIELD EXTENSIONS 1
}

\section{NICKOLAS HEEREMA ${ }^{2}$ AND DAVID TUCKER}

ABSTRACT. Let $K \supset k$ be fields having characteristic $p \neq 0$. The following is proved. If $K$ is algebraic over $k$ then $K$ is modular over $k$ if and only if $K=S \otimes_{k} M$ where $S$ is separably algebraic over $k$ and $M$ is purely inseparable, modular. If $K$ is finitely generated over $k$ (not necessarily algebraic), then $K$ is modular over $k$ if and only if $K$ $=M \otimes_{k} S$ where $M$ is finite, purely inseparable, modular over $k$, and $S$ is a finitely generated, separable, extension of $k$. This leads immediately to the representation $K=\left(S \otimes_{k} M\right) \otimes_{S} R$ where $S$ is finite separable over $k, M$ is finite, purely inseparable, modular over $k$ and $R$ is a regular finitely generated extension of $S_{0}$. This last representation dis. plays subfields of $K / k$ related to recently obtained Galois theories. The above results are used to analyze transitivity properties of modularity.

I. Introduction. Throughout this paper $K \supset k$ will be fields of characteristic $p \neq 0$. We will use the convention $K / k$ is separable, for example, to indicate that $K$ is a separable extension of $k_{0} K / k$ is modular if $K^{p^{r}}$ and $k$ are linearly disjoint over $K^{p^{r}} \cap k$ for all $r \geq 0$. This definition is due to Sweedler [10, p. 404]. He observed that separable extensions are modular and a purely inseparable extension having a finite exponent is modular if and only if (1) $K$ is a tensor product of simple extensions of $k$, or (2) $k$ is the field of constants of a set of finite and bounded rank higher derivations on $K$. For definitions see [10].

The literature contains few additional results on modular extensions and these deal essentially with purely inseparable extensions. However, there is ample reason to conclude that modularity is an important property of field extensions in general, especially in connection with Galois theories as we explain below.

Sweedler's paper referred to above was followed by a number of Galois theories for purely inseparable extensions in which the Galois extensions are modular [1]-[5], [7]. To emphasize the importance of modularity for field extensions which may not be purely inseparable, we mention the following. Heerema has shown that the classical separable theory and a purely

Presented to the Society, April 29, 1974; received by the editors July 22, 1974 and, in revised form, December 16, 1974.

AMS (MOS) subject classifications (1970). Primary 12F99.

Key words and phrases, Modular extension, linear disjointness, separability, tensor product.

1 The authors wish to thank the referee for valuable suggestions.

2 Nickolas Heerema received partial support from NSFGP 33027-X while engaged in this research. 
inseparable theory in which the maps are higher derivations combine in a natural way to form a Galois correspondence in which the Galois extensions $K$ of $k$ are normal, modular, algebraic and have finite exponent, that is, $k\left(K^{p^{n}}\right) / k$ is separable for some positive integer $n$ [6]. If $\mathcal{H}$ is any set of maps consisting of automorphisms and higher derivations of either finite or infinite rank, or both, all acting on a field $K$, then $K$ will be modular over the subfield of $\mathcal{H}$ invariants. Sweedler's proof for the purely inseparable case suffices to prove this more general statement [10, pp. 403-404]. The automorphisms in $\mathcal{H}$ need not have finite orbits.

Thus, in this paper, we consider the structure of modular extensions with a minimum of additional restrictions. Simply stated the main result is that modular extensions split along natural lines. For example, Theorem 6 states in part that an algebraic extension $K / k$ is modular over $k$ if and only if $K=S \otimes_{k} M$ where $S / k$ is separable and $M / k$ is purely inseparable mod. ular. The same theorem asserts a similar result in the nonalgebraic case in which, however, $K / k$ is finitely generated. Corollary 7 goes a step further by stating that if $K / k$ is finitely generated, then $K / k$ is modular if and only if $K=\left(S \otimes_{k} M\right) \otimes_{S} R$ where $S / K$ is finite separable, $M / k$ is finite, purely inseparable, modular, and $R / S$ is finitely generated, separable, and $S$ is algebraically closed in $R$, that is $R / S$ is finitely generated, regular.

We conclude with an analysis of transitivity properties of modularity.

II. Structure of modular extensions. Throughout this paper a separable extension $K$ of $k$ is a field extension with the property that $K$ and $k^{-1}$ are linearly disjoint over $k$ [8, Definition 3, p. 166]. Also, throughout, we make tacit use of the following result.

Proposition [8, Theorem 21, Part (1), p. 197]. Let $K \supset M \supset k$ and $K \supset$ $E \supset k$ where $E / k$ is separable and $M^{\prime} k$ is purely inseparable. The subfield of $K$ generated by $M$ and $E$ is $M \otimes_{k} E$.

We begin with a proposition which generalizes a familiar result $[8$, p. 50$]$. p. 50].

Proposition 1. Let $K \supset M \supset k$ be fields with $K / M$ separably generated and $M / k$ purely inseparable. Then there is an intermediate field $S$ separably generated over $k$ such that $K=M \otimes_{k} S_{\text {。 }}$

Proof. Let $T$ be a separating transcendency basis for $K$ over $M$ and let $S$ be the separable algebraic closure of $k(T)$ in $K$. The subfield of $K$ generated by $M$ and $S$ is $M \otimes_{k} S$. An element $\alpha$ in $K$ is separably algebraic over $M(T)$ and, hence, is separably algebraic over $M \otimes_{k}$ S. On the other hand $\alpha$ is purely inseparable over $S$ and, hence, over $M \otimes_{k} S$. Thus $a \in M \otimes_{k} S$. 
The results cited in the introduction follow directly from the above proposition and the following easily proved lemmas.

Lemma 2. If $K$ is a modular extension of $k$, then either $K$ is a separable extension of $k$ or $k^{p^{-1}} \cap K \neq k$.

Proof. If (i) $k^{p^{-1}} \cap K=k$ and (ii) $K^{p}$ and $k$ are linearly disjoint over $K^{p} \cap k$, then $K^{p}$ and $k$ are linearly disjoint over $k^{p}$, that is $K / k$ is separable.

Lem ma 3. If $K$ is a modular extension of $k$, then $K$ is also modular

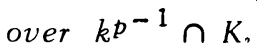

Proof. If $K / k$ is modular, then by the $p$ th root map, $K^{p^{n}}$ and $k^{p^{-1}}$ are linearly disjoint over $K^{p} p^{n} \cap k^{-1}$ for all $n \geq 0$. Since $K^{p^{n}} \cap k^{p-1} \subset K \cap$ $k^{p^{-1}} \subset k^{p^{-1}}$, it follows that $K p^{n}$ and $K \cap k^{p^{-1}}$ are linearly disjoint over their intersection $K^{p^{n}} \cap k^{-1}$ for all $n \geq 0$.

Lemma 4. Let $M=k^{p^{-\infty}} \cap K$ be the maximal subfield of $K$ purely inseparable over $k$. Then $K$ is modular over $k$ if and only if $K / M$ and $M / k$ are modular. If $K / M$ is modular, $K / M$ is separable.

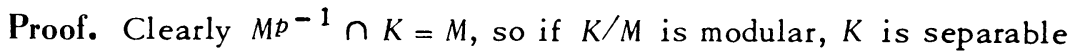
over $M$ by Lemma 2. If, in addition, $M / k$ is modular, then $K / k$ is modular by Lemma 5 below. Conversely, if $K / k$ is modular, then by Lemma $3, K$ is modular over $k p^{-n} \cap K$ for all $n$ and, hence, is modular over $M=$ $\bigcup_{n}\left(k p^{-n} \cap K\right)$ because linear disjointness is a property of finite character. But then, as noted above, $K / M$ is separable, in which case $M / k$ is modular by Lemma 5 below.

We have frequent use for the following result due to Sweedler which we state for the reader's convenience. In the reference, separable means separable algebraic, however, Sweedler's proof suffices for the more general statement given here.

Lemma 5 [10, Lemma 5, Parts 2 and 3, p. 407]. Given fields $L \supset K \supset$ $k$. If $L$ is separable over $K$, then $L$ is modular over $k$ if and only if $K$ is modular over $k$. If $L$ is modular over $k$ and $K$ is separable algebraic over $k$, then $L$ is modular over $K$.

Theorem 6. Let $K \supset k$ be fields.

(a) If $K$ is algebraic over $k$, then $K$ is modular over $k$ if and only if $K=S \otimes_{k} M$ where $M / k$ is purely inseparable modular and $S / k$ is separable algebraic.

(b) If $K$ is finitely generated over $k$, then $K$ is modular over $k$ if and 
only if $K=S \otimes_{k} M$ where $M / k$ is finite purely inseparable modular and $S / k$ is finitely and separably generated.

Proof. If $K$ splits over $k$ as in the theorem with $M / k$ modular, then, by Lemma $5, K$ is modular over $k$ in each case. Conversely, if $K$ is modular over $k$ we apply Lemma 4 , and Proposition 1 to conclude that $K$ is the indicated tensor product over $k$. That $S / k$ is not only separable but separably generated in part (b) follows from Mac Lane's criterion [8, p. 164].

If $k$ is the invariant subfield for a set of maps consisting of automorphisms and higher derivations on $K$, then $K / k$ is modular. $K$ is regular over the subfield $h$ of constants for a set of infinite higher derivations, i.e., $K / h$ is separable and $h$ is algebraically closed in $K$ [7]. $K$ is purely inseparable and modular over the constant subfield of a set of higher derio vations of bounded rank and $K$ is normal separable algebraic over the invariant field of a set of automorphisms having finite orbits. An indication of how all these subfields may fit together is given by

Corollary 7 . If $K / k$ is finitely generated, then $K$ is modularover $k$ if and only if $K=\left(S \cdot \otimes_{k} M\right) \otimes_{S} R$ where $S / k$ is finite, separable, $M / k$ is finite, purely inseparable, modular and $R / S$ is finitely generated and regular.

Proof. If $K / k$ is modular, then, by Theorem $6, K=R \otimes_{k} M$ where $M / k$ is as above and $R / k$ is finitely generated separable. Let $S$ be the algebraic closure of $k$ in $R$. Then $R / k$ separable implies $R / S$ is separable and, hence, regular. $R \otimes_{k} M=\left(S \otimes_{k} M\right) \otimes_{S} R$.

The converse is immediate by Theorem 6 .

Let $S$ and $H$ be, respectively, the separable algebraic closure and the algebraic closure of $k$ in $K$ and let $M=k^{-\infty} \cap K$. We have

$$
k \subset M \subset H \subset K \text { and } k \subset S \subset H \subset K \text {. }
$$

Theorem 8. If $K / k$ is modular, then each field in the chains $(*)$ is modular over each subfield in the chain. Conversely, if $M / k$ and $K / M$ are modular, then $K$ is modular over $k$. If $K / k$ is finitely generated and both $H / k$ and $K / H$ are modular, then $K / k$ is modular.

Proof. Since $K{ }^{p^{n}} \supset H^{p^{n}} \supset H^{p^{n}} \cap k=K^{p^{n}} \cap k$, it follows that if $K / k$ is modular, then $H / k$ is modular and, hence, by Lemma $5, H / S$ is modular. $K / M$ is separable by Lemma 4 so $H / M$ is separable. Also, by Lemma 4, $M / k$ is modular. $K / H$ is separable since $H / M$ is separable algebraic and $K / S$ is modular by the last sentence of Lemma 5. The second sentence of Theorem 8 is part of Lemma 4. If $K / H$ is modular then, by Lemma $2, K / H$ is separable and if $H / k$ is modular, $K / k$ is modular by Lemma 5.

The above theorem asserts a transitivity-like property of modularity. 
We conclude with additional observations regarding transitivity of modularity. Since there are purely inseparable extensions which are not modular $[8$, Exercise 6, p. 196], and since every exponent one purely inseparable extension is modular, it follows that, in the purely inseparable case, modularity is not transitive. An exceptional extension, that is, an algebraic, inseparable, extension $K$ of a field $k$ such that $k^{p^{-1}} \cap K=k$ provides another example of intransitivity of the modularity property. If the degree of $k$ over $k^{p}$ is greater than $p$, then $k$ will have exceptional extensions [9]. If $K / k$ is an exceptional extension, we may assume it to have exponent one. Then, if $S$ is the separable closure of $k$ in $K$, we have $K / S$ modular and $S / k$ modular and again $K / k$ fails to be modular. Theorem 6 and the following result imply that in the algebraic case the failure of a modular extension of a modular extension to be modular is always due to one of the two cases mentioned above.

Proposition 9. Let $K=S \otimes_{k} M$ with $S / k$ separable algebraic and $M / k$ purely inseparable. If $K$ is modular over $S$ then $K$ is modular over $k$.

Proof. We need the following.

Lemma 10. $S p^{-r} \cap K=S \otimes_{k}\left(k^{p^{-r}} \cap M\right)$.

Proof Clearly $S p^{-r} \cap\left(S \otimes_{k} M\right) \supset S \otimes_{k}\left(k^{p^{-r}} \cap M\right)$. Let $\{s\}$ be a basis for $S$ over $k$. Then $\left\{s_{a}^{p^{r}}\right\}$ is also a basis for $S$ over $k$. Let $x$ in $S \otimes_{k} M$ be such that $x^{p^{r}} \in S$. Then $x=\Sigma m_{\alpha} s_{\alpha}$ for some finite subset $\left\{m_{\alpha}\right\}$ of $M$ and $\Sigma m_{a}^{p^{r}} s_{a}^{p^{r}}=\Sigma h_{a} s_{a}^{p^{r}}$ with $h_{a} \in k$. Since $S$ and $M$ are linearly disjoint over $k$, we have $m_{a}^{p^{r}}=h_{a}$ for all $\alpha$ or $x \in S \otimes_{k}\left(k^{p^{-r}} \cap M\right)$.

Since $S / k$ is separable algebraic, $K / M$ is separable and, hence, modular. It follows from Lemma 4 that we need only show that $M / k$ is modular. So let $\alpha_{1}, \ldots, \alpha_{n}$ in $M$ be linearly independent over $k^{-r} \cap M$. Then $a_{1}, \ldots$, $\alpha_{n}$ are linearly independent over $S \otimes\left(k^{p^{-r}} \cap M\right)$ which, by Lemma 10 , is $S^{p^{-r}} \cap K$. Since $K / S$ is modular by hypothesis, it follows that $\alpha_{1}, \ldots, \alpha_{n}$ are linearly independent over $S^{p^{-r}} \supset k^{p^{-r}}$. Thus $M$ is modular over $k$ and the proposition is proved.

\section{REFERENCES}

1. S. U. Chase, On inseparable Galois theory, Bull. Amer. Math. Soc. 77 (1971), 413-417. MR 43 \#3237. print).

2. - Infinitesimal group scheme actions on finite field extensions (Pre-

3. R. L. Davis, A Galois theory for a class of purely inseparable field extensions, Dissertation, Florida State University, Tallahassee, Fla., 1969.

4. - A Galois theory for a class of purely inseparable exponent two field extensions, Bull. Amer. Math. Soc. 75 (1969), 1001-1004. MR 39,\#5524.

5. M. Gerstenhaber and A. Zaromp, On the Galois theory of purely ins eparable field extensions, Bull. Amer. Math. Soc. 76 (1970), 1011-1014. MR 42 \#1806. 
6. N. Heerema, A Galois theory for inseparable field extensions, Trans. Amer. Matb. Soc. 154 (1971), 193-200. MR 42 \#4527.

7. N. Heerema and J. Deveney, Galois theory for fields $K / k$ finitely generated, Trans. Amer. Math. Soc. 189 (1974), 263-274.

8. N. Jacobson, Lectures in abstract algebra. Vol. III: Theory of fields and Galois theory, Van Nostrand, Princeton, N. J., 1964. MR 30 \#3087.

9. H. F. Kreimer and N. Heerema, Modularity vs, separability for field extensions, Canad. J. Math. (to appear).

10. M. E. Sweedler, Structure of inseparable extensions, Ann. of Math. (2) 87 (1968), 401-410. MR $36 \# 6391$.

DEPARTMENT OF MATHEMATICS, FLORIDA STATE UNIVERSITY, TALLAHASSEE, FLORIDA 32306 